Wert der Konstante $K$ zusammengestellt. Beim Monde ist hier die Volumenarbeit wie bei den Planeten auf die Sonne bezogen.

\begin{tabular}{|c|c|c|c|c|c|}
\hline & Venus & Erde & Mars & Jupiter & Mond \\
\hline$M$ & I $: 408000$ & I $: 333470$ & $I: 3093500$ & I : 1047.355 & $0.0124: 33347^{\circ}$ \\
\hline$n$ & 0.8853 & I & 0.7177 & 0.2353 & 0.62 \\
\hline$a$ & 0.72333 & I & I. 52369 & 5.20280 & I \\
\hline$\gamma$ & $1.9 \mathrm{I} 3$ & I & 0.4307 & 0.03694 & I \\
\hline $2 r$ & 0.9746 & I & 0.5307 & I 1.3367 & 0.273 \\
\hline$g$ & 0.862 & I & 0.3828 & 2.477 & 0.167 \\
\hline$\hat{U}$ & $23^{\mathrm{h}} 57^{\mathrm{m}} 36^{1 / 4} \mathrm{~s}$ & $23^{\mathrm{h}} 5^{6^{\mathrm{m}}} 4^{\mathrm{s}}$ & $24^{\mathrm{h}} 37^{\mathrm{m}} 22^{\mathrm{s}} \cdot 7$ & $9^{\mathrm{h}} 5^{\mathrm{m}}$ & $27^{d} \cdot 32166$ \\
\hline$\delta$ & $14^{\circ}$ & $23^{\circ} 30^{\prime}$ & $25^{\circ}$ & $3^{\circ} 6^{\prime}$ & $3^{\circ} 30^{\prime}$ \\
\hline $\operatorname{og} K$ & 6.3343 & 6.3343 & $6.33^{62}$ & $6.33^{6} 5$ & 6.3389 \\
\hline
\end{tabular}

Bei Merkur, Uranus, Neptun ist die Berechnung von $K$ wegen Unkenntnis der Rotationselemente nicht möglich. Saturn gibt mit den gewöhnlichen Annahmen über $M$ und $n$ einen abweichenden Wert von $K(\log K=7.5287)$. Hier macht die Gestalt des Planeten wahrscheinlich eine Modifikation der Formel nötig.

Fine bildliche Darstellung der in die Formel eingehenden Größen gibt die Figur auf Tafel 5 .

Gleiwitz, ig I i im November.

H. Nies, Ingenieur.

\title{
Petit dispositif pour le pointage rapide des microscopes.
}

En vue de faciliter la tâche parfois pénible des observateurs en distances polaires, pendant les grandes soirées d'observation, nous avons imaginé un petit dispositif, s'adaptant à n'importe quel cercle et permettant de faire mouvoir les 6 microscopes à la fois dans les grands déplacements des fils.

L'appareil se compose de 6 tiges formant hexagône et reliées entre elles par des rotules à la Cardan (voir la figure, table 5).

Chacune d'elle est placée parallèlement à l'axe d'un microscope et tourne dans 2 petits coussinets en bronze $c c^{\prime}$ fixés aux rayons du faux-cercle. Elle porte en outre un pignon assez grand $P$, en bronze, également engrenant avec un autre pignon $P^{\prime}$, plus petit, placé sur l'axe du microscope.
Une manivelle $M$ placée à l'un des bouts des tiges permettrait à l'observateur de faire rapidement des de placements de 2, 3, 4 et même 5 tours comme dans les déterminations des valeurs de $\mathrm{I}^{\mathrm{t}}$ de la vis et cela pour les 6 microscopes à la fois.

La rigidité de l'ensemble est obtenue par des bagues fixés aux tiges $\mathrm{r}$ et 6 et tournant à l'intérieur et contre des coussinets.

Comme on le voit l'appareil très simple et peu coûteux serait d'une installation facile et permettrait par le petit gain de temps qu'il apporterait à chaque observation, d'éviter à l'observateur la fatigue et l'enervement des operations rapidement répétées et cause parfois des petites erreurs de visée.

rg I I Novembre.

E. Simon, Employé scientifique à l'Observatoire de Paris.

Photographic Observations of minor planets.

\begin{tabular}{|c|c|c|c|c|c|}
\hline Planet & 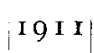 & Gr.M.T. & $\alpha$ I9II.O & $\delta$ I 9 I I.० & $\mathrm{M}$ \\
\hline 26 Prose & $\begin{array}{c}\text { Nov. } \\
\text { I } 8\end{array}$ & I $2^{\mathrm{h}} 12^{\mathrm{m}} \cdot 5$ & $2^{\mathrm{h}} 54^{\mathrm{m}} 53^{\mathrm{s}}$ & $+17^{\circ} 4^{\prime}$ & \\
\hline 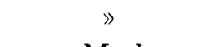 & 22 & I I 25.0 & $\begin{array}{lll}2 & 5 \mathrm{I} & 1\end{array}$ & $+\mathrm{I} 65 \mathrm{I}$ & \\
\hline 2 I 2 Medea & 22 & 1428.0 & $473^{8}$ & +2724 & \\
\hline 305 Gordonia & I 3 & 1636.5 & $334 \quad 30$ & +1627 & \\
\hline$》$ & I 5 & 1320.0 & $33^{2} 5^{6}$ & +1617 & \\
\hline 532 Herculina & I 6 & I 38.5 & 336 & $\begin{array}{lll}-- & \text { I } & 28\end{array}$ & \\
\hline
\end{tabular}

Winchester, Mass., I 9 I I Dec. I 6 .

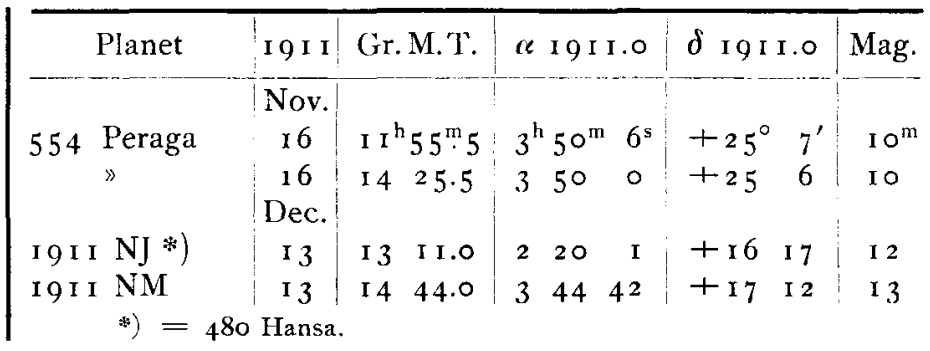

Foel H. Metcalf

480 Hansa. Dr. $V$. Drapczynski (Agram) schreibt, Paris, I9 I I Jan. 4: "Der Planet I9 I I NJ scheint mit 480 Hansa identisch zu sein. Für die Tage I9 1 I Okt. 24, Nov. r 3, 25, 26 ergibt sich zwischen Beobachtung von NJ und Berechnung für 480 Hansa (Elemente Giacobini, Ephemeride A. N. I90.14r) die mittlere Differenz - I I $^{\mathrm{m}}+8^{\prime}$. Für I9 I I Nov. ${ }^{3} .5 \mathrm{~m}$. Z. Berlin ergeben die Elemente $\alpha$ vera $=2^{\mathrm{h}} 4^{\mathrm{m}} 7^{\mathrm{s}}, \delta$ vera $=+22^{\circ}{ }_{1}^{\prime}{ }^{\prime} 7, \log r=0.4033, \log A=0.1894 . "$ Die oben mitgeteilte Beobachtung von $\mathrm{NJ}$ in Winchester Dez. I 3 bestätigt die Identität.

Wolfscher Komet. (Telegr. aus Bouzaréah vom 9. Dez. früh): Comète Wolf bien visible. Gr. I $2^{\mathrm{m}} \cdot 5$. Gonnessiat.

Inhalt zu Nr. 4546. B. Wanach. Über die Genauigkeit interpolierter und extrapolierter Uhrkorrektionen und Gänge. I69. - H. Nies. Über eine Gesetzmäßigkeit der Planetenrotation. I89. - E. Simon. Petit dispositif pour le pointage rapide des microscopes. 191. - F. H. Metcalf. Photographic Observations of minor planets. I9 I. - 480 Hansa. 191. - Wolfscher Komet. I9I. 